\title{
Proyección de cambios en la temperatura superficial del mar del Golfo de California y efectos sobre la abundancia y distribución de especies arrecifales
}

\section{Forecasting changes in sea surface temperature in the Gulf of California, and theirs effects on abundance and distribution of reef species}

\author{
Arturo Ayala-Bocos ${ }^{1,2 *}$, Héctor Reyes-Bonilla², Luis E. Calderón-Aguilera ${ }^{3}$, Ma. Dinorah \\ Herrero-Perezrul ${ }^{4}$ y Pedro C. González-Espinosa ${ }^{5}$
}

\section{RESUMEN}

El Golfo de California es una zona de importancia por su alta biodiversidad y por ser clave para las pesquerías artesanales en México. Existe preocupación de las amenazas que existen sobre él, como el calentamiento global, que ha causado cambios en las comunidades. Por esto, se pretendió estimar el posible impacto del incremento de la temperatura oceánica sobre especies claves. Se estimaron los efectos potenciales del incremento de la temperatura sobre especies arrecifales. Para realizar esta estimación, se obtuvo información de la temperatura superficial oceánica (NOAA), se construyó un modelo de tendencias de cambio de temperatura. Los datos biológicos se obtuvieron de censos visuales de seis regiones del Golfo entre el 2005 y el 2007. Posteriormente se realizó un modelo lineal por pasos (stepwise), con él se proyectaron cambios en las abundancias y distribución de los organismos. Se estima que para el año 2050 la temperatura promedio se eleve $0.63^{\circ} \mathrm{C}$; además, especies de importancia económica reducirán notablemente su abundancia al sur de los $25^{\circ} \mathrm{N}$. Por último, modelamos la respuesta de 20 especies de peces más abundantes del Golfo para estimar cambios a escala comunitaria. La simulación indica desequilibrios en la función y estructura de las comunidades. El calentamiento oceánico provocará un desbalance en la composición y función ecológica de los ecosistemas por medio de alteraciones graduales en las abundancias de las especies. Por eso, la puesta en práctica de programas de monitoreo de las comunidades sería una herramienta fundamental para detectar efectos futuros del calentamiento.

Palabras claves: Nicho ecológico, cambio global, ecología poblacional, diagnóstico pesquero, macroecología.

\footnotetext{
Ecosistemas y Conservación: Proazul Terrestre A.C. Camineros 196, Fraccionamiento del Sol, La Paz, B.C.S. México. CP 23076; artboc@yahoo.com*

2 Universidad Autónoma de Baja California Sur. Departamento de Biología Marina. Apartado postal 19-B, CP 23080. La Paz, B.C.S. México. Tel. (612) 123-8800, ext., 4160. Fax: (612) 123-8819; hreyes@uabcs.mx

3 Centro de Investigación Científica y de Educación Superior de Ensenada. Departamento de Ecología Marina. Carretera Ensenada - Tijuana 3918, CP 22860. Ensenada, B.C. México; leca@cicese.mx

4 Centro Interdisciplinario de Ciencias Marinas, Instituto Politécnico Nacional. Avenida Instituto Politécnico Nacional S/N. Colonia Playa Palo de Santa Rita. CP 23096. La Paz, B.C.S. México; dherrero@ipn.mx

5 Centro de Investigaciones Biológicas del Noroeste S. C. Programa de Estudios de Posgrado. Km 1 Carretera a San Juan de la Costa, El Comitan. Apdo. Postal 128. La Paz, B.C.S. México. CP 23097; bm.pedro.gonzalez@hotmail.com
}

Recibido: 27 de abril de 2015

Corregido: 15 de junio de 2015

Aceptado: 23 de octubre de 2015

DOI: http://dx.doi.org/10.15359/revmar.8-1.2

Rev. Mar. Cost. ISSN 1659-455X. Vol. 8 (1): 29-40, Enero-Junio 2016. 


\begin{abstract}
The Gulf of California is important because of its high biodiversity and because it is a key area for artisanal fisheries in Mexico. There are current concerns regarding threats to the Gulf, such as global warming, that have caused major changes in marine communities. Consequently, the aim of this study was to estimate the possible impact of increasing ocean temperature on key species. Potential effects of such increase on reef species are thus estimated in this paper. In order to prepare this estimate, sea surface temperature (SST) was obtained from the NOAA database, and a temperature change trend model was constructed. Biological data was obtained from visual censuses of six regions within the Gulf between 2005 and 2007. A stepwise linear model was used to forecast potential changes in species abundance and distribution. It is estimated that by 2050 average temperature will increase $0.63^{\circ} \mathrm{C}$, causing abundance of species of economic value to significantly decrease south of $25^{\circ} \mathrm{N}$. Finally, the response of the 20 most abundant fish species in the Gulf was modeled to estimate changes at community structure level. The simulation shows an imbalance in the function and structure of assemblages. Clearly, increasing sea temperature will bring an imbalance in the composition and the ecological function of the reef systems gradually changing the relative abundances of species. Therefore, the implementation of effective monitoring programs of communities would be an essential tool to detect future effects of global warming.
\end{abstract}

Keywords: Ecological niche, global change, population ecology, fishery diagnosis, macroecology.

\section{INTRODUCCIÓN}

El Golfo de California es un mar interior localizado entre la Península de Baja California y los estados de Sonora y Sinaloa, al noroeste de México. Alberga más de 800 especies de peces y aproximadamente 4500 de invertebrados (Brusca et al. 2005), además de ser un área clave de gran importancia para las pesquerías artesanales en México (Rodríguez-Quiroz et al. 2010). La región presenta múltiples ecosistemas costeros, incluyendo planicies arenosas, manglares, mantos de rodolitos, estuarios y arrecifes rocosos y coralinos (Lluch-Cota et al. 2007). En especial, estos últimos han recibido una particular atención científica y comercial por representar zonas de alta riqueza de especies (Roberts et al. 2002).

El crecimiento económico de los estados costeros del Golfo de California ha incrementado de manera notable las actividades comerciales y turís- ticas, causando un aumento en el nivel de perturbaciones antropogénicas a los ecosistemas (Enríquez-Andrade et al. 2005). Por ello, aun cuando esta es una de las regiones marinas mejor conservadas en el Pacífico Oriental Tropical y con un gran número de programas de conservación, existe una gran preocupación por el futuro al considerar potenciales agentes de contaminación costera, modificaciones de los hábitats, sobrepesca y sobrepasarse la capacidad de carga de los sitios por turismo excesivo (Aguilar et al. 2007). Además, el Golfo de California no es inmune a los efectos que podría causar el cambio climático global. El Panel Intergubernamental sobre Cambio Climático (2014) menciona que en los últimos 100 años la temperatura superficial global (terrestre y oceánica) promedio se ha incrementado entre $0.65^{\circ} \mathrm{C}$ y $1.6^{\circ} \mathrm{C}$, que el nivel del mar 
ha subido de 0.17 a $0.21 \mathrm{~m}$ desde inicios del siglo pasado (1900). En el ámbito global, la temperatura superficial del océano se ha incrementado entre 0.09 y $0.13^{\circ} \mathrm{C}$ por década en los últimos 40 años. Diferentes modelos muestran que la temperatura superficial (terrestre y oceánica) podría ascender entre $0.3^{\circ} \mathrm{C}$ y $4.8^{\circ} \mathrm{C}$ para finales de siglo (2081-2100), y que el nivel del mar podría aumentar entre 0.26 y $0.85 \mathrm{~m}$ (IPCC, 2014). Como resultado, se han reportado cambios en la distribución y composición de comunidades de especies marinas en zonas templadas y subtropicales (Albouy et al. 2012; Pont et al. 2015). Existe una particular preocupación por lo que podría ocurrir en las comunidades por el blanqueamiento coralino generado por el incremento en la temperatura oceánica (Hoegh-Guldberg, 1999; Baker et al. 2008; Logan et al. 2014; Li \& Reidenbach, 2014), aparentemente causando una fuerte disminución de abundancia de corales en el mundo. Para el Golfo de California solo hay información anecdótica (e.g., Reyes-Bonilla, 2003), dada la carencia de largas series de datos biológicos o de información oceanográfica detallada. Sin embargo, el uso de información espacial a gran escala puede ayudar a abordar este problema al permitir construir modelos de tolerancia fisiológica de las especies, a partir de los cuales se puede predecir su distribución (Smith et al. 2008). Algo cierto es que las condiciones serán distintas en el futuro, razón por la que se deben realizar investigaciones para prever el impacto que tendría este fenómeno sobre la distribución, abundancia y riqueza de las diferentes especies de importancia ecológica y comercial. Este tipo de herramientas macroecológicas tienen una enorme aplicación para pronosticar el impacto que puede tener el calentamiento global sobre las comunidades marinas, y abren la posibilidad de proveer recomendaciones para mejorar la planeación estratégica de los creadores de planes de manejo y tomadores de decisiones. Desde esta óptica, el objetivo del trabajo fue evaluar la tendencia de cambio de la temperatura superficial del mar en el Golfo de California entre 1983 y el 2014, y posteriormente emplear los resultados como insumos para proyectar modificaciones en la distribución y abundancia de algunas especies relevantes.

\section{MATERIALES Y MÉTODOS}

El Golfo de California se ubica al noroeste de México. Es un mar interior de forma alargada, orientado de noroeste a sureste. Tiene una longitud aproximada de $1500 \mathrm{~km}$ y en su parte más amplia tiene $205 \mathrm{~km}$ de ancho en la zona sur y una mínima de $117 \mathrm{~km}$ a la mitad del Golfo. La Península de Baja California Sur es el límite oeste, el macizo continental del noroeste de México es su límite este, al norte se encuentra con la desembocadura del río Colorado, al sur, el límite es una línea imaginaria que se extiende desde la punta sur de la Península en Cabo San Lucas $\left(23^{\circ} \mathrm{N}\right)$ hasta Cabo Corrientes, Jalisco, México $\left(20^{\circ}\right.$ N) (Rusnak et al. 1964; Álvarez-Borrego, 1983; Thomson et al. 2000). 
Es importante hacer mención que la información oceanográfica para construir los modelos es hasta el 2007 y no a la fecha, para que coincidieran con la información de datos biológicos (censos visuales de organismos), la cual es de 2004 a 2007.

La primera parte de la investigación consistió en construir una base de datos oceanográficos que sustentara los modelos de cambios biológicos. El Golfo fue dividido en cuadrículas de $1^{\circ} \times 1^{\circ}$ de latitud-longitud $(\mathrm{N}=27), \mathrm{y}$ para cada una se obtuvieron registros superficiales (promedios y valores máximos y mínimos anuales) de salinidad (UPS), nitratos, silicatos y fosfatos (micromol) y de concentración de clorofilas $\left(\mathrm{mg} / \mathrm{m}^{3}\right)$. La información provino del World Ocean Atlas (2015; valores de 1950-2007) y de imágenes de satélite del Ocean Color Web, 2015 (datos de 1987 a 2007). Adicionalmente, para conocer las tendencias de cambio de la temperatura superficial del Golfo se tomaron los promedios mensuales de cada cuadrícula de $1^{\circ} \times 1^{\circ}$ entre 1983 y el 2006 (NOAA, 2013; 2015), y se calculó su promedio anual. Dichos valores se incluyeron en una regresión lineal simple que utilizó el año como factor de prueba y donde la pendiente representa la tasa de elevación anual de la temperatura en el sitio (Neter et al. 1997). Por último, con la ecuación de regresión extrapolamos los valores hasta el año 2050, con el fin de obtener pronósticos anuales de temperatura $\mathrm{y}$ plantear el posible escenario.

Para el caso de los datos biológicos, se hizo uso de una serie temporal de registros (2005-2007) que consta de censos visuales (500 censos en total aproximadamente) en transectos de banda de $20 \mathrm{~m} \times 5 \mathrm{~m}$ para peces y de 20 $\mathrm{m} \times 2 \mathrm{~m}$ para invertebrados, realizados por integrantes del laboratorio de Sistemas Arrecifales de la Universidad Autónoma de Baja California Sur (entre ellos, todos los autores de este trabajo). Se tomaron en cuenta principalmente especies claves en seis regiones arrecifales rocosas de la Península de Baja California (Bahía de Los Ángeles, $28^{\circ} \mathrm{N}$; Santa Rosalía, $27^{\circ} \mathrm{N}$; Loreto, $26^{\circ} \mathrm{N}$; La Paz, $24^{\circ} \mathrm{N}$; Cabo Pulmo, $23^{\circ}$ $\mathrm{N}$, y Los Cabos, $22^{\circ} \mathrm{N}$ ).

Del total de los censos realizados, se extrajeron los datos de abundancia de la cabrilla sardinera Mycteroperca rosacea (Streets, 1877), el pez ángel de Cortés Pomacanthus zonipectus (Gill, 1862) y el pepino de mar café Isostichopus fuscus (Ludwig, 1875), con el fin de trabajarlos de manera individual. Las tres especies tienen una gran importancia económica y se explotan en al menos dos de los estados costeros del Golfo (SAGARPA, 2010); además de que las dos últimas también están dentro de la Norma Oficial Mexicana, en la categoría de Protección Especial (Diario Oficial de la Federación, 2010). Para modelar los factores que determinan su abundancia usamos una regresión lineal por pasos ("stepwise"), con la rutina "ridge", que evita introducir variables correlacionadas en el modelo (Neter et al. 1997). Se aplicaron los datos de temperatura, salinidad y nutrientes como factores para predecir la abundancia 
actual de las tres especies en cada región estudiada frente a la Península de Baja California, y se creó un modelo que seleccionó las variables que más afectan la abundancia. Luego se predijeron las abundancias de las especies por zona a partir únicamente de los datos oceanográficos. El último análisis estadístico se dirigió a la evaluación del posible efecto del calentamiento del océano sobre las especies indicadas. Para ello, se sustituyó en la ecuación de regresión el valor del coeficiente de la temperatura, ya sea de forma directa o indirecta. En este caso, con los resultados de los modelos se crearon escenarios de cambio local de las abundancias causado por el aumento de temperatura, en $+1^{\circ},+2^{\circ} \mathrm{y}+3^{\circ} \mathrm{C}$.

\section{RESULTADOS Y DISCUSIÓN}

En la Figura 1 se muestra la tasa de elevación de la temperatura del Golfo de California en cuadrículas de $1^{\circ} \times 1^{\circ}$, en ella se nota que la zona del sur de Sonora y norte de Sinaloa (zona centro-este del Golfo de California) es donde el calentamiento está siendo más intenso, mientras que en el Canal de Ballenas (grandes islas del norte del Golfo) la pendiente de la regresión años-temperatura es casi de cero, indicando estabilidad térmica en las últimas dos décadas y media. Tomando los datos de todas las cuadrículas estimamos que para el año 2050 la elevación promedio de la temperatura superficial en el Golfo de California será de $0.63^{\circ} \mathrm{C}$, pero esto depende de la zona geográfica, pues, para el 2050 prácticamente todo el sur tendrá temperaturas medias superiores a $\operatorname{los} 25^{\circ} \mathrm{C}$ (Fig. 2).
Por otra parte, los gráficos de abundancia estimada del ángel de Cortés $(P$. zonipectus) y de la cabrilla sardinera ( $M$. rosacea) indican que el calentamiento del Golfo de California traerá aparejada una sensible disminución de los números de individuos de cada especie, y de hecho, el modelo sugiere que las poblaciones de la cabrilla desaparecerán en el centro y suroeste de la Península de Baja California si la temperatura se incrementa $3^{\circ} \mathrm{C}$ (Fig. 3). Revisando las ecuaciones de regresión, tales cambios tienen su origen en que las dos especies tienen afinidad con aguas frías y con zonas de alta productividad, variables que se verán afectadas por el calentamiento en el futuro, información que coincide con la distribución actual de ambas especies, donde sus mayores abundancias se presentan en zonas de surgencias y aguas templadas (Díaz-Uribe et al. 2001).

De manera semejante, el análisis de las tendencias posibles de cambio en el pepino café (Isostichopus fuscus) muestra, que las poblaciones pueden disminuir de forma considerable si las temperaturas se incrementan más de $1{ }^{\circ} \mathrm{C}$ (Fig. 4). Las poblaciones del pepino café tienen una alta relación con la temperatura oceánica, y los periodos de reproducción están fuertemente ligados a los cambios de temperatura (HerreroPerezrul et al. 1999), por lo que cambios considerables de temperatura superficial del mar podrían ocasionar cambios en la población. En adición, según el modelo, la zona sur de la Península sufrirá una disminución superior al 30\% de la población de este organismo, si la temperatura se incrementa 3 grados. 


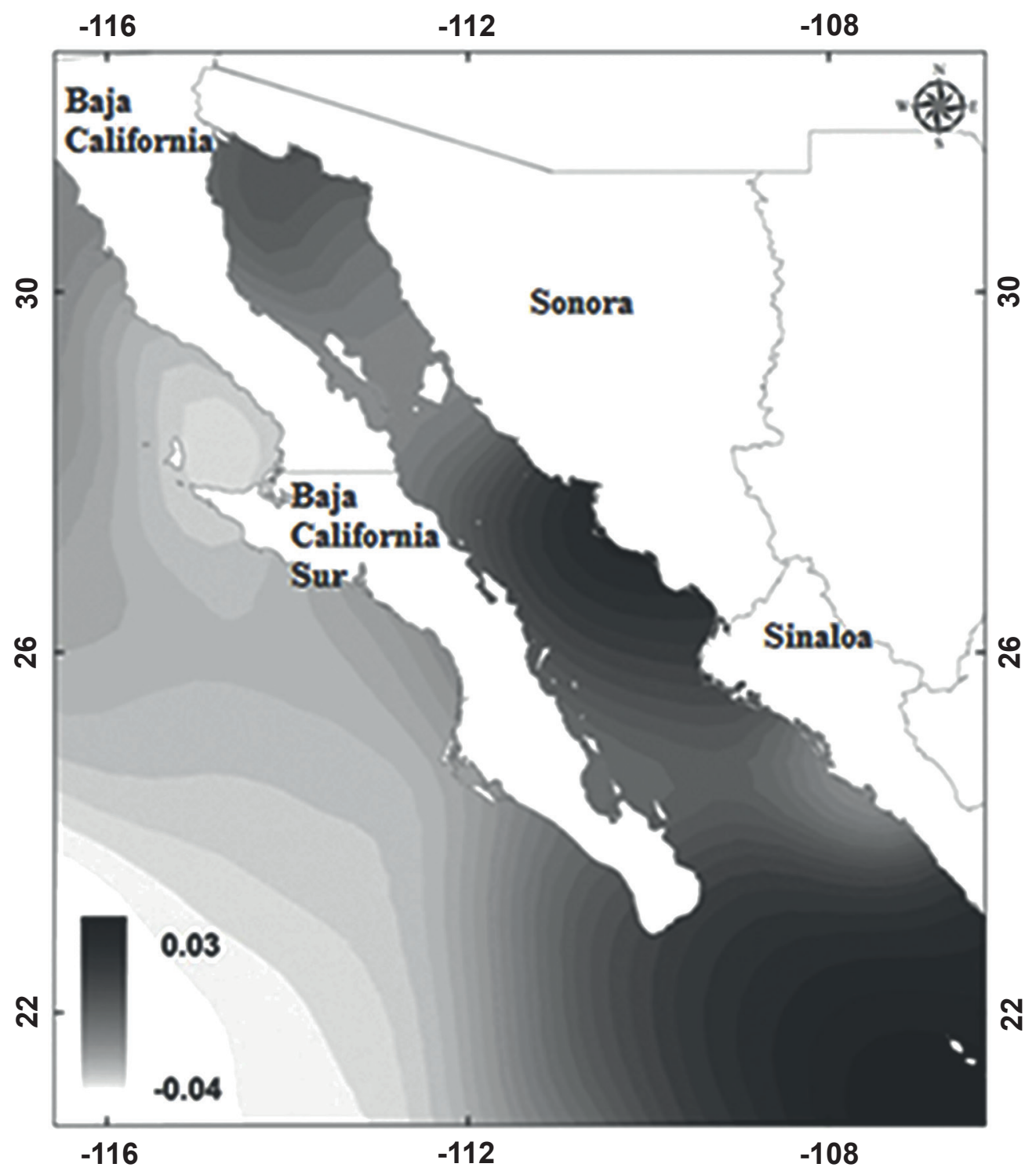

Fig. 1. Tendencias en la elevación de la temperatura del Golfo de California, en grados centígrados por año (1982-2006)

Fig. 1. Trends in temperature elevation of the Gulf of California in Celsius per year (1982-2006)

Ello sugiere que la pesca de este recurso sería impactada desproporcionadamente siendo más considerable la disminución de este recurso en Baja California Sur con respecto a Baja California.
Finalmente, efectuamos un análisis de la respuesta de las 20 especies más abundantes en las siete zonas arrecifales de interés en el Golfo de California (listado taxonómico en Ayala-Bocos \& Re- 


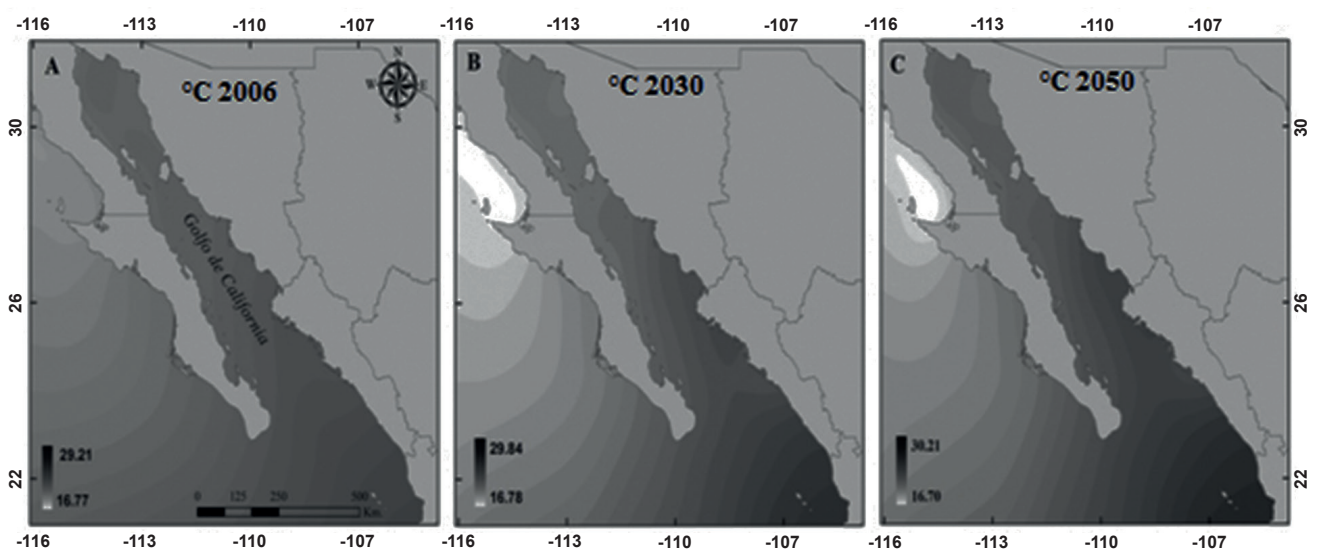

Fig. 2. Temperatura superficial promedio en el 2006 del Golfo de California (en grados centígrados; izquierda) y pronóstico para el 2030 (centro) y el 2050 (derecha)

Fig. 2. Average surface temperature in 2006 in the Gulf of California (in Celsius, left) and forecast for 2030 (center) and 2050 (right)
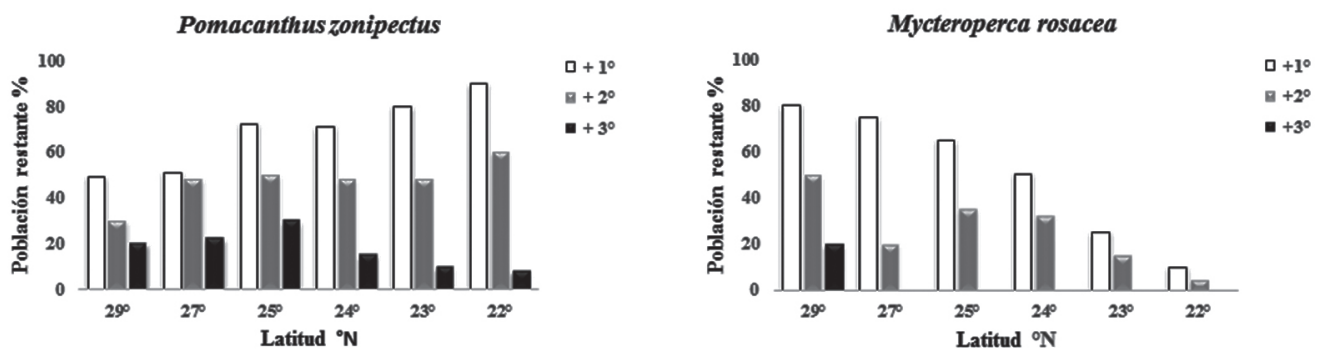

Fig. 3. Proyección de cambios en la abundancia y distribución de Pomacanthus zonipectus (izquierda) y Mycteroperca rosacea (derecha) en la Península de Baja California, según el aumento de la temperatura

Fig. 3. Forecasted changes in abundance and distribution of Pomacanthus zonipectus (left) and Mycteroperca rosacea (right) in Baja California, according to temperature increase

yes-Bonilla, 2009). Como se observa en la Figura 5, las asociaciones actuales se agruparon en el lado izquierdo del dendrograma. Sin embargo, una vez que la temperatura asciende la posición de las estaciones dentro de las ramas se modifica de forma desordenada y no gradual. Nuestra conclusión es que dadas las diferencias en los niveles de tolerancia al cambio ambiental, cada especie mos- trará comportamientos específicos y no necesariamente congruentes con los del resto. Tales variaciones idiosincrásicas implican dos aspectos claves: a) el calentamiento del mar traerá un fuerte desbalance en la composición y, por ende, en la función ecológica de los sistemas arrecifales del Golfo de California; y b) no es de esperarse que las modificaciones sean detectables por medio de ex- 


\section{Isostichopus fuscus}

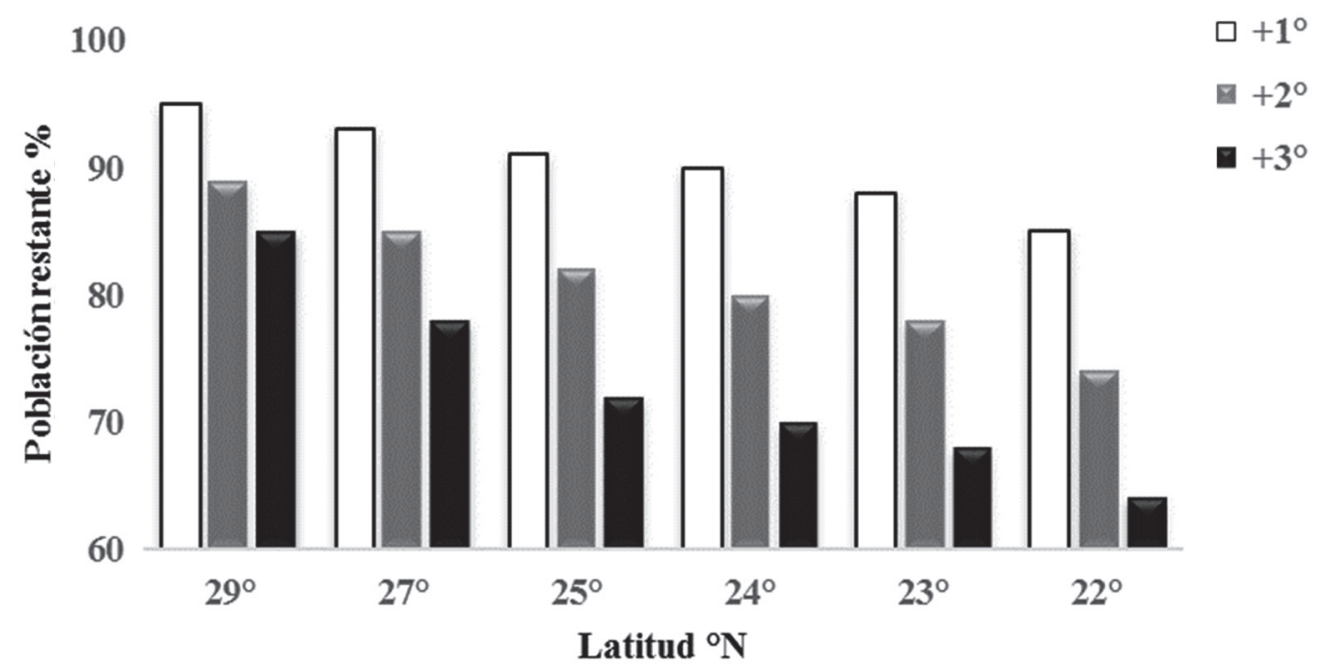

Fig. 4. Proyección de cambios en la abundancia y distribución de Isostichopus fuscus en la Península de Baja California, según el aumento de la temperatura

Fig. 4. Forecasted changes in abundance and distribution of Isostichopus fuscus in Baja California, according to temperature increase

tinciones locales o cambios abruptos de composición de las comunidades, sino más bien serán alteraciones graduales en las abundancias relativas de las especies. Este desbalance en las comunidades por el aumento de la temperatura oceánica, ya se ha registrado en otros lugares del mundo, ocasionando efectos similares (Perry et al. 2005; McKenzie et al. 2007; Albouy et al. 2012).

Considerando esta situación, recomendaríamos la puesta en práctica de programas de monitoreo efectivos de las comunidades y de los niveles poblacionales de las especies claves, como una herramienta fundamental para detectar los efectos futuros del calentamiento.

Tanto organizaciones gubernamentales como no lucrativas buscan generar políticas para balancear el aprove- chamiento de los recursos naturales con la protección de la calidad ambiental. Desde esta perspectiva, los modelos de cambio climático y espacio de nicho, y las predicciones resultantes acerca del estado futuro de las poblaciones y ecosistemas, son fundamentales para ofrecer escenarios probables, los cuales sirvan como prevención y guía en la toma de decisiones. Además, el ofrecer este tipo de información a la sociedad en general (una vez mejor validada o más completa) permitiría que los diversos actores ponderen y planeen sus propias actividades actuales y futuras, maximizando el impacto hacia la conservación. En resumen, las herramientas ejemplificadas pueden convertirse en insumos claves, para apoyar el manejo y la conservación del Golfo de Cali- 


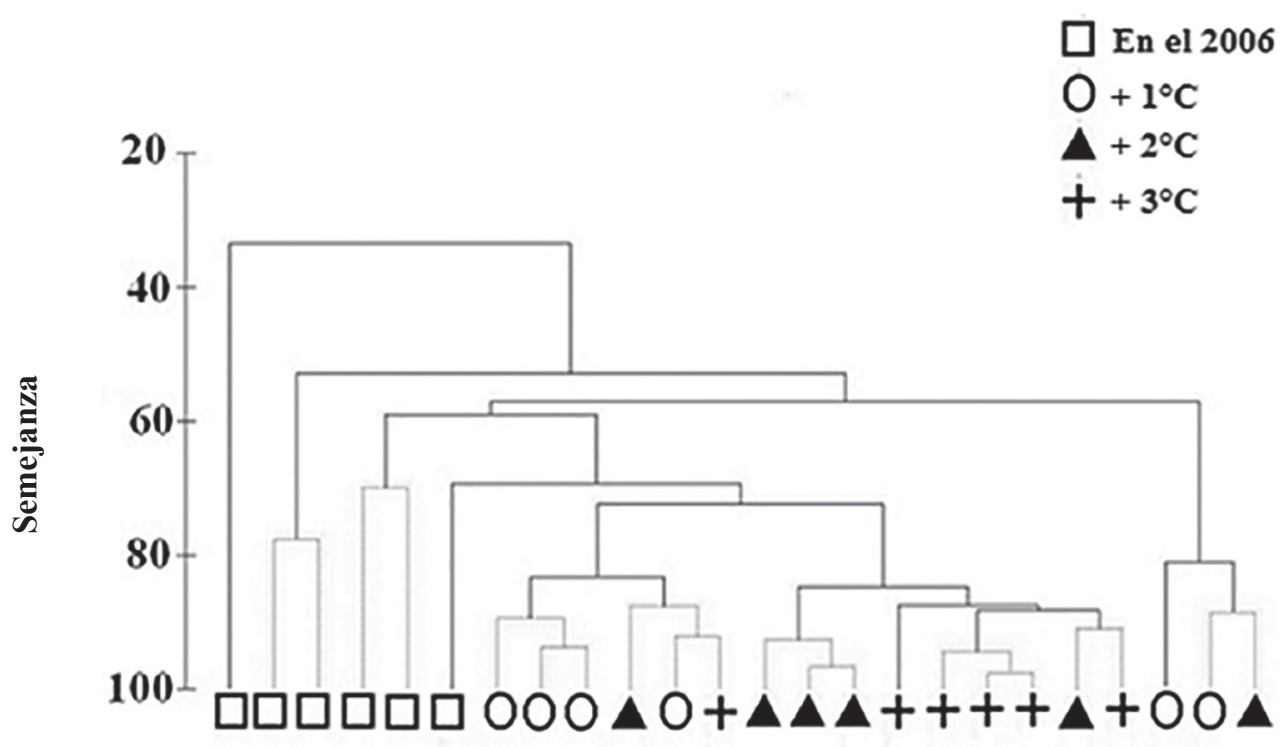

Agrupamiento de escenarios de 6 comunidades del Golfo de California

Fig. 5. Dendrograma de agrupamiento que muestra el grado de semejanza (coeficiente de Bray-Curtis) entre las comunidades de seis zonas arrecifales del Golfo de California, en el 2006 (cuadros), y los predichos por modelos de elevación de temperatura de $1^{\circ} \mathrm{C}$ (círculos), $2^{\circ} \mathrm{C}$ (triángulos) y $3^{\circ} \mathrm{C}$ (cruces)

Fig. 5. Cluster dendrogram showing the degree of similarity (Bray-Curtis coefficient) between six coral reef communities in the Gulf of California for 2006 (squares) and cluster predicted by models of temperature increase of $1{ }^{\circ} \mathrm{C}$ (circles), $2^{\circ} \mathrm{C}$ (triangles) and $3^{\circ} \mathrm{C}$ (crosses)

fornia, y fortalecer su aplicabilidad. La aplicación de estos modelos para proyectar la vulnerabilidad a la extinción de especies o cambios de distribución se ha convertido en una herramienta potencialmente útil para informar las decisiones de gestión de recursos. Los modelos pueden ser más útiles para la identificación de oportunidades de conservación reconociendo nuevos hábitats disponibles o si algún hábitat actual ya no existirá en virtud de futuros cambios climáticos (Schwartz, 2012).

Los resultados presentados en este trabajo son un primer intento formal y cuantitativo para denotar cambios po- tenciales en la distribución y abundancia de especies marinas en el noroeste de México. Las proyecciones son aún imprecisas y deberán ser revisadas con información oceanográfica y biológica actualizada en los años por venir, es decir, contar con planes de monitoreo permanentes y continuos. Sin embargo, constantemente se trabaja en el desarrollo y mejoramiento de manera económica, social y ecológica para los residentes del litoral del Golfo de California, los cuales deberán ser discutidos y priorizados por las autoridades, con el fin de mejorar y crear estrategias alternas para el manejo y la toma de decisio- 
nes. Es precisamente por la posibilidad de ofrecer contextos específicos por lo que los alcances del enfoque macroecológico son importantes. En este trabajo se presentó un intento formal de cómo realizar estos modelos y su uso, pero además del ejemplo aquí mostrado es posible desarrollar modelos con otro tipo de variables e información, ya que los datos requeridos para efectuar esta clase de modelos están ya disponibles, gracias a la existencia de series temporales largas de estadísticas pesqueras, monitoreos en zonas protegidas y registros oceanográficos generados por agencias gubernamentales y no gubernamentales, tanto nacionales como internacionales, facilitando así, el realizar este tipo de modelos para crear herramientas y estrategias de manejo.

Sin duda alguna, la amalgama de esfuerzos por conocer el funcionamiento de los ecosistemas del Golfo en distintas escalas de tiempo y espacio rendirá frutos y mejorará nuestras posibilidades de conservar esta importante región del Pacífico Oriental.

\section{AGRADECIMIENTOS}

Agradecemos a Saúl González por su participación en la elaboración de este artículo, así como a Gabriela Díaz Erales (Fondo Mexicano para la Conservación de la Naturaleza) por la elaboración de los mapas. Asimismo, agradecemos al Instituto Nacional de Ecología por permitirnos presentar esta investigación en la Bienal del Golfo de California. Finalmente, agradecemos a los tres revisores anónimos por las observaciones realizadas a este trabajo.

\section{BIBLIOGRAFÍA}

Aguilar, V., Hernández, D. \& Kolb, M. (2007). Análisis de vacios y omisiones en conservación de la biodiversidad marina de México. México: CONABIO/ CONANP/TNC/PRONATURA.

Albouy, C., Guilhaumon, F., Araujo, M. B., Mouillot, D. \& Leprieur, F. (2012). Combining projected changes in species richness and composition reveals climate change impacts on coastal Mediterranean fish assemblages. Global Change Biol., 18, 2995-3003. http://dx.doi. org/10.1111/j.1365-2486.2012.02772.x

Álvarez-Borrego, S. (1983). Gulf of California. In C. B. H. Ketchum (Ed.), Estuaries and Enclosed Seas (pp. 427-449). Amsterdam, Netherlands: Elsevier.

Ayala-Bocos, A. \& Reyes-Bonilla, H. (2009, Julio). Analysis of reef fish abundance in the Gulf of California, and projection of changes by global warming. Ponencia presentada para el XI Simposio Internacional de Arrecifes de Coral, Fort Lauderdale, EE. UU. Baker, A. C., Glynn, P. W. \& Riegl, B. (2008). Climate change and coral reef bleaching: An ecological assessment of long-term impacts, recovery trends and future outlook. Estuar. Coast. Shelf. Sci., 80, 435-471. http://dx.doi. org/10.1016/j.ecss.2008.09.003

Brusca, R. C., Findley, L. T., Hastings, P. A., Hendrickx, M. E., Torre Cosío, J. \& Van der Heiden, A. M. (2005). Macrofaunal diversity in the Gulf of California. In J. L. E. Cartron, G. Ceballos \& R. S. Felger (Eds.), Biodiversity, ecosystems and conservation in northern Mexico (pp. 179-202). Oxford, England: Oxford University Press.

Diario Oficial de la Federación. (2010). Secretaría de Agricultura, Ganadería, Desarrollo Rural, Pesca y Alimentación 
(SAGARPA). Carta Nacional Pesquera. Recuperado en julio 20, 2005, disponible en http://www.conapesca.sagarpa. gob.mx/wb/cona/actualizacion_de_la_ carta_nacional_pesquera_2010

Díaz-Uribe, J. G., Elorduy-Garay, J. F. \& González-Valdovinos, M. T. (2001). Age and growth of the Leopard Grouper, Mycteroperca rosacea, in the southern Gulf of California, Mexico. Pacific Sci., 55(2), 171-182. http:// dx.doi.org/10.1353/psc.2001.0012

Enríquez-Andrade, R., Anaya-Reyna, G., Barrera-Guevara, J. C., Carvajal-Moreno, M. A., Martínez-Delgado, M. E., Vaca-Rodríguez, J. \& Valdés-Casillas, C. (2005). An analysis of critical areas for biodiversity conservation in the Gulf of California. Oc. Coast. Manag., 48, 31-50. http://dx.doi.org/10.1016/j. ocecoaman.2004.11.002

Herrero-Perezrul, M. D., Reyes-Bonilla, H., García-Domínguez, F. \& CintraBuenrostro, C. E. (1999). Reproduction and growth of Isostichopus fuscus (Echinodermata: Holothuroidea) in the southern Gulf of California, Mexico. Mar. Biol., 135, 521-532. http://dx.doi. org/10.1007/s002270050653

Hoegh-Guldberg, O. (1999). Climate change, coral bleaching and the future of the world's coral reefs. Mar. Fresh. Res., 50, 839-866. http://dx.doi. org/10.1071/MF99078

IPCC. (2014). Climate Change 2014: Synthesis report. Geneva, Switzerland: Intergovernmental Panel on Climate Change.

Li, A. \& Reidenbach, M. A. (2014). Forecasting decadal changes in sea surface temperatures and coral bleaching within a Carribean coral reef. Coral Reefs., 33(3), 847-861. doi: 10.1007/s00338014-1162-1. http://dx.doi.org/10.1007/ s00338-014-1162-1
Lluch-Cota, S. E., Aragón-Noriega, E. A., Arreguin-Sánchez, F., Aurioles-Gamboa, D., Bautista-Romero, J. J., Brusca, R. C., Cervantes-Duarte, R., ... \& Sierra-Beltran, A. P. (2007). The Gulf of California: review of ecosystem status and sustainability changes. Prog. Oceanogr., 73, 1-26. http://dx.doi. org/10.1016/j.pocean.2007.01.013

Logan, C. A., Dunne, J. P., Eakin, M. \& Donner, S. D. (2014). Incorporating adaptive responses into futures projections of coral bleaching. Global Change Biol., 20(1), 125-139. http://dx.doi. org/10.1111/gcb.12390

McKenzie, B. R., Gislason, H., Möllmann, C. \& Köster, F. (2007). Impact of $21^{\text {st }}$ century climate change on the Baltic Sea fish community and fisheries. Global Change Biol., 13(7), 1348-1376. http://dx.doi. org/10.1111/j.1365-2486.2007.01369.x

Neter, J., Kutner, M., Nachtsheim, C. \& Wassermann, W. (1997). Applied linear statistical models $\left(4^{\text {th }}\right.$ ed). New York, EE. UU.: McGraw-Hill.

NOAA. (2013). National Weather Service. Reynolds SST Analysis. Recuperado en marzo 25, 2006, disponible en http:// www.nhc.noaa.gov/aboutsst.shtml

NOAA. (2015). National Centers for Environmental Information World Ocean Atlas. Recuperado en marzo 21, 2006, disponible en www.nodc.noaa.gov/OC5/ SELECT/woaselect/woaselect.html

Ocean Color Web. (2015). Ocean Color Feature. Recuperado en mayo 15, 2006, disponible en http://oceancolor.gsfc.nasa.gov Perry, A. L., Low, P. J., Ellis, J. R. \& Reynolds, J. D. (2005). Climate Change and Distribution Shifts in Marine Fishes. Science, 308(5730), 1912-1915. http:// dx.doi.org/10.1126/science.1111322 
Pont, D., López, M., Carrel, G., Rogers, C. \& Haidvogl, G. (2015). Historial change in fish species distribution: shifting reference conditions and global warming effects. Aquat Sci., 77, 441-453. http://dx.doi.org/10.1007/ s00027-014-0386-z

Reyes-Bonilla, H. (2003). Coral reefs of the Pacific coast of Mexico. In J. Cortés (Ed.), Latin American coral reefs (pp. 313-330). Amsterdam, Netherlands: Elsevier. http://dx.doi.org/10.1016/ b978-044451388-5/50015-1

Roberts, C. M., McClean, C. J., Veron, J. E. N., Hawkins, J. P., Allen, G. R., McAllister, D. E. \& Werner, T. B. (2002). Marine biodiversity hotspots and conservation priorities for tropical reefs. Science, 295, 1280-1284. http://dx.doi. org/10.1126/science.1067728

Rodríguez-Quiroz, G., Aragón-Noriega, E. A., Valenzuela-Quiñónez, W. \& EsparzaLeal, H. (2010). Artisanal fisheries in the conservation zones of the Upper Gulf of California. Rev. Biol. Mar. Oceanogr.,
45(1), 89-98. http://dx.doi.org/10.4067/ S0718-19572010000100008

Rusnak, G. A., Fisher, R. L. \& Shepard, F. P. (1964). Bathymetry and Faults of Gulf of California. In T. H. Van Andel \& G. G. Jr. Shor. (Eds.), Marine Geology in the Gulf of California (pp. 5975). Tulsa, EE. UU.: American Association of Petroleum Geologist.

Schwartz, M. W. (2012). Using niche models with climate projections to inform conservation management decisions. Biol. Cons., 155, 149-156. http://dx.doi. org/10.1016/j.biocon.2012.06.011

Smith, F. A., Lyons, S. K., Morgan-Ernest, S. K. \& Brown, J. H. (2008). Macroecology: more than the division of food and space among species on continents. Prog. Phys. Geog., 32, 115-138. http://dx.doi. org/10.1177/0309133308094425

Thomson, A. D., Findley, L. T. \& Kerstich, A. N. (2000). Reef fishes of the sea of Cortez. Texas, EE. UU.: The University of Texas Press. 